\title{
The Complete System of Linear Complexes and one Quaternary Quadric.
}

\author{
By P. N. Das Gupta and Professor H. W. Turnbull.
}

(Received 2lst October 1929. Read 8th June 1929.)

$\S 1$. In the following pages we give a complete system of projective concomitants for any number of linear complexes and one quadric, in a quaternary field. The investigation follows the earlier ${ }^{1}$ paper on mixed quaternary forms, in which the corresponding system, omitting the quadric, was given. Such a system, for one quadric and one linear complex was given ${ }^{2}$ by Weitzenböck who used complex symbols. A detailed investigation of the case of two linear complexes and a quadric, together with their geometry, has been given elsewhere. ${ }^{3}$

Our ground forms are first a quadric $f$, denoted in the usual symbolic notation by

$$
\Sigma a_{i j} x_{i} x_{j}=a_{x}{ }^{2}=a_{x^{2}}^{\prime}, \quad(A p)^{2}, \quad u_{a}{ }^{2} \ldots \ldots \ldots \ldots \ldots \text { (1) }
$$

where $A=a a^{\prime}, p=u v$, and $(A p)^{2}, u_{a}^{2}$ denote line and plane forms of the quadric originally given in point variables $x$.

Secondly there are any number of linear complexes,

where

$$
(K p),(L p),(M p),(N p),(Q p),(R p)
$$

$(K p)=K_{12} p_{34}+K_{13} p_{42}+K_{14} p_{23}+K_{34} p_{12}+K_{42} p_{13}+K_{23} p_{14} \ldots \ldots$

with similar expansions for the others. We shall speak of elements $u, p, x, a, A, \alpha, K, L, \ldots, R$, to denote these variables and symbols indifferently. They are classified by their currencies 1, 2, 3:

$$
\left.\begin{array}{l}
\text { Currency } 1 ; u, a, a^{\prime}, b, c, \\
\text { Currency } 2 ; p, A, K, L, \ldots R, \\
\text { Currency } 3 ; x, a, \alpha^{\prime} .
\end{array}\right\}
$$

${ }^{1}$ H. W. Turnbull, Proc. London Math. Soc., 2, 25 (1926), 303-327.

2 Crelle 137, (1910), 65-82.

$\because$ By P. N. Das Gupta in a paper to appear in the Proc. Lond. Math. Soc. (1930). 
The higher currency may be resolved into the lower: for example

$$
x=u v w=p w, \quad p=u v, \quad A=a a^{\prime}, \quad a=A a^{\prime \prime}, \text { etc. }
$$

More precisely $x_{i}=(u v w)_{j k l} ; i, j, k, l=1,2,3,4$ in some order.

\section{The Prepared System.}

§2. Every polynomial concomitant can be symbolized by products of factors consisting of thirteen types ${ }^{1}$; and when all varieties of the elements (3) are substituted in these types, we obtain 40 elementary forms, called the prepared system. These can be written down at once, grouped as sixteen concomitants, twenty factors each involving one quadric symbol $a, A$ or $a$, and four involving two such symbols.

The sixteen concomitants.

$u_{x},(p p), a_{a}^{2},(K L),(K K),(K p),(u K L x),(a K L p a)$

(aKLpa), (uKLMu), (xKLMx), (aKLMa), (aKLMa), $(u K L M N x)$,

$(K L M N Q R)-(N Q R K L M)=\left|K_{14} L_{24} M_{34} N_{23} Q_{31} R_{12}\right|$, $(K L M N Q p)-(N Q p K L M)=\left|K_{14} L_{24} M_{34} N_{23} Q_{31} p_{12}\right|, \mid$

The twenty factors of type $\{a\},\{A\},\{a\}$

$\{a\} \quad a_{x},(a K u),(a K L x),(a K L M u),(a K L M N x),(a K L M N Q u)$,

$\{A\} A p,(A K),(u A K x),(u A K L u),(x A K L x),(u A K L M x)$, $(A K L M N Q),(A K L M N p)$,

$\left.\{a\} u_{a},(\alpha K x),(\alpha K L u),(a K L M x),(a K L M N u),(\alpha K L M N Q x).\right)$

The four factors of type $\{a, a\}$

(apKa), (aKLa), (apKLMa), (aKLMNa).

In forming the above list, elements of odd currency are called the end elements-for obvious reasons. They manifestly furnish factors such as $(u \ldots u),(u \ldots a),(u \ldots x),\left(a \ldots a^{\prime}\right),(a \ldots a)$, ete. According to the general theory of prepared systems for quadrics, $\left(a \ldots a^{\prime}\right)$ is equivalent to $(a \ldots a)$, and therefore can be rejected. We also exclude factors involving $A$ simultaneously with itself or with $a$ or $a$, and cases of $p$ simultaneously with another variable.

2 H. W. Turnbull, loc. cit. 312. 
For example: $(a K A L u) \equiv-(a A K L u)=-(\alpha K L u)$, on making use of the fundamental identity (loc. cit. p. $311(22)$ )

$$
(\lambda K A i)+(\lambda A K i)+(A K)(\lambda i)=0 .
$$

The sign $\equiv$ as usual denotes equivalence of forms when reducible terms are dropped.

§3. Grade of a factor. The intermediate elements $p, A, K$, etc. in a factor can always be deranged among themselves, as in a determinant, with the exception of the factors $(p p),(K p),(K K),(K L)$, $(A p),(A K)$. The derangement may produce reducible terms, but they are negligible. For example, by this identity (8),

$$
(u A K L M x)=-(u A L K M x)-(K L)(u A M x) .
$$

Hence $(u A K L M x)$ and $(u A L K M x)$ are considered equivalent, (their sum is reducible); and evidently any pair of $A, K, L, M$ may be interchanged.

The number of intermediate elements in a factor is called the grade.

Expansion of a factor. Each factor has a total currency equal to $4 \nu$, a multiple of four : and the factor can be expanded as a sum of terms each consisting of $\nu$ original factors provided by the fundamental theorem. For example.

$$
\begin{aligned}
& (a K L M N Q u)=\Omega_{l, n}(a K l)\left(l^{\prime} M n\right)\left(n^{\prime} Q u\right), \\
& (K L M N Q R)=\Omega_{k, m, q}\left(k^{\prime} L m\right)\left(m^{\prime} N q\right)\left(q^{\prime} R k\right),
\end{aligned}
$$

where $K=k k^{\prime}, L=l l^{\prime}$, etc. The symbol $\Omega$ denotes determinantal permutation of the letters indicated by its suffix. A comma between suffixes isolates two separate permutations. $\Omega_{l, n}$ denotes $2 \times 2$ terms, and $\Omega_{k, m, q} 2 \times 2 \times 2$ terms, because each suffix, such as $l$, indicates a permutation of two letters $l l^{\prime}$, giving rise to two terms, say $\phi\left(l, l^{\prime}\right)$ and $-\phi\left(l^{\prime}, l\right)$.

It is convenient to denote a product of factors, at any stage, by $g_{1} g_{2} g_{3} \ldots g_{\nu}$. For instance, applied to (10), the direction "transfer $u$ from $g_{3}$ to $g_{1}$ " would indicate a fundamental identity of the type

$$
(a b c d)(u \ldots)=(u b c d)(a \ldots)-(a u c d)(b \ldots)+\ldots, \ldots \text {. } \ldots \text { (12) }
$$

which replaces the right member of (10) by

$$
\begin{aligned}
& \Omega_{l, n}\left[(u K l)\left(l^{\prime} M n\right)\left(n^{\prime} Q a\right)+(a K u)\left(l^{\prime} M n\right)\left(n^{\prime} Q l\right)\right] \\
& +\Omega_{l, n, k}\left(a u k^{\prime} l\right)\left(l^{\prime} M n\right)\left(n^{\prime} Q k\right) .
\end{aligned}
$$




\section{Fundamental Identities}

$\S 4$. Besides the identity (12) and its dual, involving elements $\alpha, \beta, x$ in factors $(\alpha \beta \gamma \delta)(x \ldots)$, we shall frequently need the identity (8) which deranges adjacent intermediate elements of a factor, and also a related identity which can be written

$$
(i K \bar{B} \lambda)=(i \bar{B} K \lambda)+(i \lambda)(\bar{B} K)
$$

where $\vec{B}=\beta \gamma$. Here we make the following three definitions

(i) $(i K \bar{B} \lambda)=(i K \cdot \beta \gamma \cdot \lambda)=(i \beta)(K \gamma \lambda)+(i \gamma)(K \lambda \beta)+(i \lambda)\left(K \beta_{\gamma}\right)$,

(ii) $(i \bar{B} K \lambda)=(i \cdot \beta \gamma \cdot K \lambda)=(i \beta)(\gamma K \lambda)-(i \gamma)(\beta K \lambda)$,

(iii) $(\bar{B} K)=(\beta \gamma K)=\left(\beta K_{\gamma}\right)=(k \beta)\left(k^{\prime} \gamma\right)-\left(k^{\prime} \beta\right)(k \gamma)$.

Identity (13) follows immediately on these definitions.

In (8) and (13) $i$ denotes an element (or string of elements) whose currency is $1(\bmod 4)$, and $\lambda$ one whose currency is $3(\bmod 4)$.

For example, if $\phi=(u K L a)(x M N Q \lambda)$, then we can write

$$
(u K L . a x \cdot M N Q \lambda)=(u K L a)(x M N Q \lambda)-(u K L x)(\alpha M N Q \lambda) .
$$

But $(u K L x)$ reduces the latter term. Accordingly, by (13),

$$
\begin{aligned}
\phi & \equiv(u K L \cdot \alpha x \cdot M N Q \lambda) \\
& \equiv(u K L N Q \lambda)(\alpha x M)+(u K L M . \alpha x \cdot N Q \lambda) .
\end{aligned}
$$

'This procedure, or its analogous dual, is very useful.

§5. Complete System of Concomitant Types for six Linear Complexes and a Quadric.

Seven Invariants

$a_{\alpha}{ }^{2}, \quad(K L), \quad(A K)(A L), \quad(a K L M a), \quad(\alpha K L M \alpha), \quad(A K)(K L M N Q A)$, $(K L M N Q R)-(N Q R K L M)=\left|K_{34} L_{24} M_{34} N_{23} Q_{31} R_{12}\right|$.

Eight Complexes

$(K p), \quad(A p)^{2}, \quad(A p)(A K), \quad(a K L p a), \quad(a K L p a), \quad(A p)(K L M N Q A)$, $(A K)(L M N Q p A),(K L M N Q p)-\left(N^{\top} Q p K L M\right)=\left|K_{14} L_{24} M_{34} \nu_{23} Q_{31}, p_{12}\right|$.

Grade Ten Covariants

Ten Contravariants

\begin{tabular}{l|l} 
& $A_{x}{ }^{2}(x K L M x)$, \\
2 & $(a K x)(\alpha L x),(a K L x) a_{x}$ \\
3 & $(a K L a) a_{x}(a M x)$, \\
4 & $(a K L x)(a M N x)$, \\
& $(a K L M x)(a N x)$, \\
5 & $(x K A L x)(A M)$, \\
& $(a K L a) a_{x}(\alpha M N Q x)$, \\
6 & $(\alpha K L M x)(a N Q K x)$,
\end{tabular}

$u_{a}^{2},(u K L M u)$ $(a K u)(a L u),(\alpha K L u) u_{x}$, $(a K L \alpha)(a M u) u_{a}$, $(a K L u)(a M N u)$, $(a K L M u)(a N u)$, $(u K A L u)(A M)$, $(a K L \alpha)(a M N Q u) u_{\alpha}$ $(a K L M u)(a N Q K u)$. 
Twenty-three Mixed Concomitants

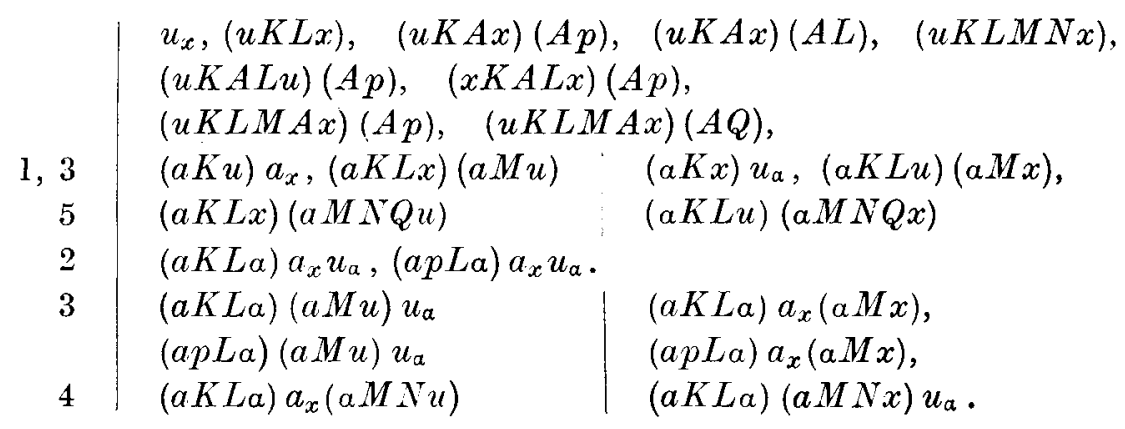

In the above complete list, the proof of which will follow, $K, L, M, N, Q, R$ denote six or less linear complexes. For example the type $(K L)$ covers the particular case $(K K)$ of an invariant of one complex. Entries in parallel columns are correlatives; and, for convenience of reference, the grades of some of the forms have been indicated. It will be seen that the grade cannot exceed six; and what follows will shew that for each grade there are several equivalent ways of writing one form.

\section{Proof of the Complete System.}

$\S 6$. If we form products of factors in the prepared system, it is manifest that the irreducible concomitants involving quadric symbols are found among the following cases :

$$
\begin{aligned}
\text { I } & \{A\}\{A\}, \\
\text { II } & \{a\}\{a\},\{a\}\{a\},\{a, a\}\{a, a\}, \\
\text { III } & \{a, a\}\{a\}\{a\} .
\end{aligned}
$$

Case $I \quad\{A\}\{A\}$.

Such forms contain two factors, each involving $A$, but no other symbol of the quadric. If the factors also involve $u$ or $x$, they may be written $(u A \ldots u),(x A \ldots u),(x A \ldots x)$; other possible factors are of grade 2 or 6 only- $(A K)$, $(A p),(A K L M N Q),(A K L M N p)$.

ThEOREM I. If $\lambda, \mu, \rho, \sigma$ are each of currency $3(\bmod 4)$ but contain no quadric symbol, $(\lambda A \mu)(\rho A \sigma)$ is reducible.

For if $A=a b$, the expansion of this product is expressible as $\Sigma\left(a_{\lambda} a_{\rho}\right)\left(b_{\mu} b_{\sigma}\right)$, where each factor is palpably a concomitant.

As each of the above factors, which involve $u$ or $x$, is of this type, only one such factor can occur in an irreducible form; and 
in this case we prove that the complementary factor cannot have grade 6 , but must be $(A K)$ or $(A p)$.

In fact

$$
(u R A x)(K L M N Q A) \equiv 0 .
$$

Proof. Write this as

$$
-2\left[\Omega_{A}(u R a)(b x)\right] \Omega_{l, n}(a K l)\left(l^{\prime} M n\right)\left(n^{\prime} Q b\right), \text { where } A=a b .
$$

Transfer $a$ from factor $g_{1}$ to factor $g_{4}$, and, in the term which breaks up $M$, convolve $N$ in $g_{4}$.

The result is

$$
\begin{array}{cc}
\Sigma(u R L K a)(b Q N M A x)-2(u R M N A x)(a K L Q b) \\
+(u R M L K a)(x A Q b) .
\end{array}
$$

Now, by identity (8), several times repeated,

$$
\begin{aligned}
(b Q N M A x)= & -(b Q N A M x)-(b Q N x)(M A) \\
= & (b Q A N M x)+(b Q M x)(N A)-(b Q N x)(M A) \\
= & -(b A Q N M x)-(b N M x)(Q A)+\text { etc. }
\end{aligned}
$$

But $b A$ is zero. Hence

$$
(b Q N M A x)=-(b N M x)(Q A)+(b Q M x)(N A)-(b Q N x)(M A) .
$$

Substituting in the first term of (16) we have

$$
\begin{aligned}
\Sigma(u R L K a) & (b Q N M A x)=-\Sigma \Sigma(u R L K a)(b N M x)(Q A) \\
& =-\frac{1}{2} \Sigma \Sigma(u R L K A N M x)(Q A) \ldots \ldots \ldots
\end{aligned}
$$

The last term of (16) can be dealt with in the same way, since

$$
\begin{aligned}
(x A Q b) & =-(x b)(A Q)-(x Q A b) \\
& =-(x b)(A Q) .
\end{aligned}
$$

In the remaining term of (16), which can be written

$$
(u R M N A x)(A K L Q) \text {, }
$$

the second factor resolves and always yields a concomitant $(K L),(L Q)$ or $(Q K)$. Thus the whole expression (16) is equivalent to a sum of terms such as

$$
(u R L K A N M x)(Q A) \text {. }
$$

Finally this sixth grade factor $(u R L K A Q N x)$ is equivalent to reducible terms ${ }^{1}$ such as

$$
(R L K A Q N) u_{x}, \quad \Sigma(u R L K A x)(Q N), \quad \sum(u R L K Q x)(A N) .
$$

\footnotetext{
1 loc. cit., p. 313 .
} 
This proves the result; and this method is also applicable to any product $(\phi)(K L M N Q A)$ where $(\phi)=(u \ldots \ldots u),(u \ldots \ldots x),(x \ldots x)$. Similarly if $Q$ is replaced by a variable $p$ in the second factor.

The only surviving forms, containing $u$ or $x$, are those already quoted in the complete system, namely

$(u K A x)(A L), \quad(u K A x)(A p), \quad(u K L M A x)(A Q), \quad(u K L M A x)(A p)$, $(u K A L u)(A M), \quad(u K A L u)(A p)$,

and duals of these last two forms.

Other forms of type $\{A\}\{A\}$ are products, two at a time, of the factors $(A K),(A p),(K L M N Q A)$ and $(L M N Q p A)$, excepting a grade 12 product which is always reducible. For such a product is equivalent to

or

$$
\begin{aligned}
& (a K L M N Q b)(a R S T U V b) \\
& (a K L M N Q . b a . R S T U V b) \bmod R,
\end{aligned}
$$

or, by shifting the $b a$ towards either end, to

$$
\Sigma(Q b a)(a K L M N R S T U V b)
$$

As before, the last factor is reducible; and this completes Case I.

\section{Cases II and III.}

$\S 7$. These contain no symbol $A$, but factors of types $(a \ldots u)$, $(a \ldots \ldots),(a \ldots \ldots),(a \ldots x),(a \ldots \ldots)$, whose grades are odd, odd, even, even, even, respectively. The odd grades may be $1,3,5$, and the even, $0,2,4$.

Theorem II. If the tolal grade in a pair of such factors exceed six, the product is reducible.

Proof. Consider $\phi=(u K L M a)(u N Q R S \lambda)$. We have $(C f . \S 4$ (14))

$$
\begin{aligned}
\phi & \equiv(u K L M . a u . N Q R S \lambda) \\
& \equiv-(u a u K L M N Q R S \lambda)+\Sigma(M a u)(u K L N Q R S \lambda) .
\end{aligned}
$$

The string of six consecutive elements like $K L M N Q R$ in each term, implies a reducing factor $(K L)$ or $(K L M N Q R)$, since the symbol $A$ is absent from among them; and this reduces $\phi$. This or a similar dual argument, involving the shifting of $a x$ by use of identity (13), applies to all such products of grade exceeding six, except the cases

$$
(a \ldots x)(a \ldots x),(a \ldots u)(a \ldots u),(a \ldots a)(a \ldots a) .
$$


The only case among these of a total grade exceeding 6 is

$$
\phi=(i K L M N \lambda)(i Q R S T \lambda), \quad i=u, a ; \quad \lambda=a, x .
$$

We expand and write this

$$
\phi=\Omega g_{1} g_{2} g_{3} g_{4} g_{5} g_{6},
$$

maintaining the exact order of symbols $i, K, \ldots, \lambda$. For example $g_{5}$ is $\left(r^{\prime} S t\right)$. Let $i$ be transferred from $g_{1}$ to $g_{5}$, and in the resulting term where $S$ is broken, let $T$ be convolved in $g_{5}$. 'Hence

$$
\phi \equiv(i S T \lambda)(i Q R K L M N \lambda)-(i Q R S i)(\lambda N M L K T \lambda)+\text { etc. }
$$

Terms are either obviously reducible or contain a grade six factor and reduce.

This completes the proof of Theorem II.

\$8. It remains to examine all forms, falling under cases II and III where the total grade in two factors is six or less. For case II the last theorem limits the grade to six; and the forms are duly entered in the above tabulated Complete System. In particular for grades 1, 2, 3 no variations exist, but for grade 4 under the type $(a \ldots x)(a \ldots x)$ two possibilities arise,

$$
(a K L M N x)(a x) \text { and }(a K L x)(a M N x) \text {. }
$$

These however are equivalent; for if the expansion of the first form is $\Omega g_{1} g_{2} g_{3} g_{4}$, and $a$ is transferred from $g_{4}$ to $g_{2}$, and $L$ is convolved in the term which breaks $M$, then a sum of terms $(a K L x)(a M N x)$ is produced.

Similarly for the dual form $(\alpha K L M N u) u_{\alpha}$. For $(a \ldots a)(a \ldots a)$, grade four provides only

$$
\phi=(a K L a)(a M N a)
$$

but this is reducible.

Proof. Let $a=b c d$, resolved into three symbols all equivalent. to $a$. Then

$$
\begin{aligned}
\phi= & (b c d K L a)(b c d M N a) \\
& =-3(K b c)(L d a)(b c d M N a) \\
& \equiv \quad 3(K b c)(L d a)[(M c d)(N b a)-(M b d)(N c a)] \\
& \equiv \quad 3(K b c)(L d a)\left[\Omega_{n}(b c a n)\left(n^{\prime} M d\right)\right] \\
& \equiv-\Omega_{k}(k b c a)\left(k^{\prime} L d\right)(b c a N M d) \\
& \equiv \quad(a K L d)(a N M d) \equiv-\phi .
\end{aligned}
$$


Hence $2 \phi$ is reducible. A similar reduction holds for the grade six form ( $a K L a)$ ( $a M N Q R a)$.

Grade 5 in Case II, leads to equivalent types

$$
(a K L M N x)(a Q u) \text { and }(a K L x)(a M N Q u)
$$

and as in (16), the former reduces to the latter. Similarly for grade 6. These with their duals account for all, in Case II, that have been retained.

\section{Case III.}

$\S 8$. This alone remains to be considered. Let the typical form be written

$$
\phi=\{a, \alpha\}\{a\}\{\alpha\}=\{\lambda ; \mu, \nu\}
$$

where $\lambda, \mu, \nu$ denote the grades of the factors $\{a, \alpha\},\{a\}$ and $\{\alpha\}$ respectively. By Theorem I these integers satisfy the relations

$$
\begin{gathered}
\lambda+\mu<7, \quad \mu+\nu<7, \quad \nu+\lambda<7, \\
\lambda=2,4, \quad \mu \geqslant 0, \quad \nu \geqslant 0 .
\end{gathered}
$$

Theorem III. $\{4 ; \mu, \nu\} \equiv \Sigma\{2 ; \mu+2, \nu\} \equiv \Sigma\{2 ; \mu, \nu+2\}$.

Proof. Let $\phi=(a P Q R S a)(a \rho)(\alpha i)$.

Then $\phi \equiv(a . i a . P Q R S a)(a \rho) \bmod a_{\alpha}$,

$$
\begin{aligned}
& \equiv \Sigma \pm(i a P)(a Q R S \alpha)(a \rho)+(\alpha P Q . i a \cdot R S \alpha)(a \rho) \\
& \equiv(\alpha P Q i)(a R S \alpha)(a \rho)-(\alpha P Q a)(i R S \alpha)(a \rho) .
\end{aligned}
$$

This expresses $\phi$ as two terms of type $\{2 ; \mu+2, \nu\}$ together with reducible terms. Correlatively by use of (13) the result $\{2 ; \mu, \nu+2\}$ follows.

Theorem IV. $(a P Q \alpha)(a \rho)(\alpha R \sigma) \equiv(a P Q \alpha)(a \sigma)(\alpha R \rho)$.

Symbolically $(2 ; \mu, \nu) \equiv(2 ; \nu-1, \mu+1)$.

Proof. Resolve a into $A b$. Then

$$
\begin{gathered}
(a P Q \alpha)(a \sigma)(a R \rho)=-3(a P Q A b)(a \sigma)(A R)(b \rho) \\
\equiv-3(a A P Q b)(a \sigma)(A R)(b \rho) .
\end{gathered}
$$

Convolving $a, A$ once more this gives

$$
\begin{gathered}
(a A P Q b)(a A R \sigma)(b \rho) \\
\equiv(a P Q b)(a R \sigma)(b \rho) \equiv(a P Q \alpha)(a \rho)(a R \sigma)
\end{gathered}
$$

on replacing $b$ by $a$ and reversing the first factor and then $P Q$, by (8). 
Now consider the forms by ascending grade. By Theorem III we must have $(a P Q a)$ as factor; hence the grade is 2 or more. For grades 2 and 3 there are no alternatives to the forms in the complete list. For grade 4 we have

$$
(2 ; 2,0), \quad(2 ; 1,1), \quad(2 ; 0,2),
$$

the two latter being equivalent by Theorem IV. The former is $(a K L a)(a M N x) u_{a}$ which is equivalent to $(a K L . a x N M a) u_{a}$, and by shifting $a x$ twice it becomes $(a, a x . K L N M a) u_{a}$, yielding $a_{x}(a K L N M a) u_{a}$ which reverts to type $(2 ; 0,2)$ by theorem IV. Hence there is only one new type for grade 4.

For grade 5 we have two types

$$
(2 ; 3,0) \equiv(2 ; 1,2), \quad(2 ; 2,1) \equiv(2 ; 0,3) .
$$

The former equivalence is shewn as follows:

$$
\begin{gathered}
(a K L a)(a M N Q u) u_{a} \equiv-(a K L a)(a \cdot u a, M N Q u) \\
\equiv \Sigma(a K L a)(u a M)(a N Q u) \equiv \Sigma(2 ; 1,2) .
\end{gathered}
$$

The latter equivalence, $(2 ; 2,1) \equiv(2 ; 0,3)$, follows from Theorem IV.

Forms of grade six are reducible. For consider $(2 ; 2,2)$. We have

$$
\begin{aligned}
& (a K L a)(a M N x)(a P Q u) \equiv-(a K L \cdot a x \cdot N M a)(a P Q u) \\
& \equiv-(a \cdot a x . K L N M a)(a P Q u) \equiv a_{x}(a K L N M a)(a P Q u) \\
& \equiv-a_{x}(a K L N M \cdot a u \cdot Q P a) \equiv-a_{x}(a K L N M Q P a) u_{a}
\end{aligned}
$$

whence the invariants $(K L)$ and $(K L N M Q P)$ are precipitated, and the form reduces. The same applies to all such forms $(2 ; \mu, \nu)$ for which $2+\mu+\nu>5$.

This completes the investigation. 